\title{
评《微分方程分支理论》
}

\author{
梁肇军 \\ (华中师范大学数学系, 武汉 430079)
}

(韩茂安, 朱德明著. 北京：煤炭工业出版社, 1994. 定价 50 元)

由韩茂安、朱德明两位博士撰写的《微分方程分支理论》是一部优秀的学术著作. 从基 础理论研究的角度看, 微分动力系统的一个重要方向是刻画结构稳定性. 平面动力系统结构 稳定性的概念最早由 A. А. Андронов 和Л. С. Понтрягин 在 1937 年提出, 并在解 析系统的情况下给出结构稳定的必要充分条件, 50 年代 H. DeBaggis 减弱了条件并完成证明, 1962 年 M.M.Peixoto 又将其推广到二维流形上去.

设 $X, Z$ 是拓扑空间, 开集 $U \subset X, \Lambda$ 是某一参数空间内的一个开集, 而 $f: U \times \Lambda \rightarrow Z$ 是一个 给定的连续函数, 令 $S=\{(x, \lambda) \in U \times \Lambda: f(x, \lambda)=0\}$ 是方程 $f(x, \lambda)=0$ 的所有解集, 对固定的 $\lambda$, 令 $S_{\lambda}=\{x:(x, \lambda) \in S\}$ 是解集处的“横截面”，人们关心的是集 $S_{\lambda}$ 对 $\lambda$ 有什么依赖关系.

考虑系统 $\frac{\mathrm{d} u}{\mathrm{~d} t}-h(u, \lambda)=0$, 其中 $(u, \lambda) \in \Omega \times \Lambda$ ，而 $\Omega$ 是某个 Banach 空间 $B$ 的开集，对于从 $(0$, $\infty)$ 到 $B$ 的函数 Banach 空间 $X, Z$, 令 $U \subset X$, 而 $U=\{u \in X ; u(t) \in \Omega, t \in(0, \infty)\}$, 上述系统可 以写为 $f(x, \lambda)=0$, 其中

$$
f: U \times \ddot{E} \rightarrow Z, \quad f(u, \lambda)(t)=\frac{\mathrm{d} u(t)}{\mathrm{d} t}-h(u(t), \lambda) .
$$

设上述方程是 $\Omega$ 上的一个强连续半群 $T_{\lambda}(t), t \geqslant 0$, 两个微分方程称为是等价的, $h(\cdot, \lambda) \sim h(\cdot$, $\omega)$, 如果存在一个同胚 $g: \Omega \rightarrow \Omega$, 使得 $g$ 把 $T_{\lambda}(t)$ 的轨道映到 $T_{\omega}(t)$ 的轨道且关于时间保持 定向. 如果在 $\lambda_{0}$ 处 $h\left(\cdot, \lambda_{0}\right)$ 不是结构稳定的, 则称 $\lambda_{0}$ 为分支值. 从理论上说, 分支值就是系统结 构不稳定的参数值. 分支理论在 20 世纪后 40 年得到巨大的发展.

一般的分支理论又分为两个不同方向, 即静态与动态分支. 静态分支理论是有关函数中 一些参数改变时, 零点集合的结构所产生的变化, 本书介绍其中 Liapunov-Schmidt 这一经典 的方法，而把重点放在论述动态分支.

基础理论研究应密切关注在实际问题中的应用, 这是新一代青年学术工作者的潮流, 本 书作者们在选题时就注意了它的应用背景.

众所周知, 在物理、力学、工程、化学反应、生态学、社会经济、金融等领域的科学研 究中, 经常以微分方程(组)为其数学模型进行研究, 例如 Tamnenbaum ${ }^{[1]}$ 指出, 飞机控制方面 的研究中出现二次多项式微分系统的极限环问题.

人们注意到应用中的每个问题都含有一些物理参数, 它们可以在某些特定的集合中变动, 故了解这些参数变动时系统的定性性态是重要的. 一个设计得好的系统应该是当参数关于其 原设计值变动一个小量时系统的定性性态并不改变, 这样的数学模型归结为微分方程模型时, 就说它是结构稳定的. 然而, 当模型中的参数大幅度变动时它的性态可能要改变. 定性性质的 一种改变可能意味着原系统的稳定性的变化, 从而系统必然会取不同于原来设计的状态.

如果我们在科学实验中所取的参数值恰好使模型的定性性态剧烈改变, 这将导致实验不 
能重复再现, 其原因可能是从实际问题归结为数学模型时, 将一些主要因素当成 “次要因素” 忽略了, 也可能是人们在客观上对实际问题的复杂性尚未有足够的认识. 总之, 粗粮地说, 这 样的变化发生时的参数值就称为分支值. 寻找分支值对于完全地把握这个系统是绝对必要的, 它对于指导我们进行科学实验有重要的意义.

一个微分方程在一定条件下对应一个向量场, 动态分支就是关于向量场中一些参数变化 时对微分方程轨道的极限集结构中所产生的变化. 本书作者除了介绍较成熟的、带有局部性 质的 Andronov-Hopf 分支以外, 对一些要求用更高深的微分方程理论才能解决的困难问题, 如 周期轨道、同宿环、异宿环和不变环面等问题开展深入的研究, 获得了可喜的成果. 例如从 同宿环、异宿环分支出极限环及其个数的深刻结果, 他们先后在《中国科学》《数学学报》 发表 4 篇论文, 这些结果已总结在本书第 7,8 两章中, 并得到老一辈数学家的充分肯定(参见文 献[2, 3]).

本书对近 20 年新出现的普适开折、分支的余维、大范围分支等课题, 以及符号动力系统、 三维系统同宿分支与混沌等国际上非常活跃的问题均做了介绍.

此外, 本书还对目前尚缺少计算方法的无穷远处赤道闭轨分支出极限环的问题进行讨论, 笔者认为这是一个十分困难的问题, 至于赤道极限环的重数及判定, 仍然是一个未解决的问题.

本书的作者是我国自行培养、并且在广泛与国内外学术交流的环境中成长起来的中青年 科研工作者. 1982 年, 他们在攻读硕士学位期间, 正值美国著名教授 J. K. Hale (《微分方程杂 志》主编)和周修仪(S. N. Chow)先后到南京大学、复旦大学等校讲授分歧理论方法, 他们深 得名家教益. 以后他们在教学与科研的实践中多次到英国、意大利、美国等进行学术访问, 不 断吸收新的学术思想, 经过长期的积累而形成本书的构思.

全书立足于深刻的应用背景, 深入浅出、文笔流畅, 将读者从基础知识一直引向研究前 沿, 这在某种程度上反映了作者们的治学心得. 相信本书对广大中青年科学工作者和应用数 学工作者是很有益的.

\section{参考 文献}

Tamnenbaum A. Book Reviews. Bull Amer Math Soc, 1987, 17(1): 178 179

2 叶彦谦. 多项式微分系统定性理论. 上海: 上海科学技术出版社, 1995

3 张芷芬, 李承治, 郑志明, 等. 向量场的分岔理论基础. 北京: 高等教育出版社, 1997 